${ }^{5}$ Università degli Studi di Modena e Reggio Emilia, Dipartimento di Scienze Biomediche-Metaboliche e Neuroscienze, Modena, Italy * Corresponding author.

Introduction Metabolic syndrome (MetS) is defined by metabolic and cardio-vascular impairments and is frequently associated with anxiety and depressive disorders. Both MetS and anxietydepressive syndromes feature similar systemic inflammatory alterations. Inflammation of the large bowel is also a key factor for the development of colorectal cancer (CRC).

Objective To measure the prevalence of MetS and symptoms of anxiety and depression among patients undergoing colonoscopy. Methods Cross-sectional study. Patients undergoing colonoscopy aged 40 or more, with negative history for neoplasia or inflammatory bowel disease, were enrolled. Data collected: colonoscopy outcome, presence/absence of MetS (IDF and ATP III criteria), presence/absence of depressive and anxiety symptoms assessed with HADS.

Results The sample was made up of 53 patients (female 24, $45.3 \%$ ). Mean age was $60.66 \pm 9.08$. At least one adenoma was found to 23 patients (43.3\%). Prevalence of MetS ranged from 34\% to $36 \%$ (ATP III and IDF criteria, respectively). Prevalence of depressive and anxiety symptoms was $20 \%$ and $33 \%$, respectively.

Conclusion Prevalence of MetS, anxiety and depressive symptoms among patients undergoing colonoscopy was higher than in the general population.

Disclosure of interest The authors have not supplied their declaration of competing interest.

http://dx.doi.org/10.1016/j.eurpsy.2017.02.236

EW0623

\section{Impact of anxiety-depressive symptoms on outpatients' quality of life: Preliminary results from an Italian observational study}

S. Tassi ${ }^{1}$, G. Rioli ${ }^{2, *}$, G. Mattei ${ }^{3}$, S. Ferrari ${ }^{4}$, G.M. Galeazzi ${ }^{4}$

${ }^{1}$ Università degli studi di modena e Reggio Emilia, Corso di Laurea in Medicina e Chirurgia, Modena, Italy

${ }^{2}$ Università degli Studi di Modena e Reggio Emilia, Scuola di

Specializzazione in Psichiatria, Sassuolo, Italy

${ }^{3}$ Università degli Studi di Modena e Reggio Emilia, Scuola di Specializzazione in Psichiatria, Modena, Italy

${ }^{4}$ Università degli Studi di Modena e Reggio Emilia, Dipartimento di Medicina Diagnostica- Clinica e di Sanità Pubblica- sezione di Psichiatria, Modena, Italy

* Corresponding author.

Introduction Several studies have shown an association between the Short-Form 36 (SF36) scores and anxiety-depressive symptoms, suggesting that depression in particular could reduce Quality of Life (QoL) to the same, and even greater, extent than chronic noncommunicable diseases, such as diabetes and hypertension.

Aims To explore the relationship among QoL and anxiety, depressive and anxiety-depressive symptoms in an outpatient sample. Methods Cross-sectional study. Inclusion criteria: outpatients aged $\geq 40$ years, without history for cancer, attending colonoscopy after positive faecal occult blood test. Collected data: blood pressure, blood glucose, lipid profile. Psychometric test: Hospital Anxiety and Depression Scale (HADS). QoL was assessed with SF36. Statistics performed with STATA13.

Results 54 patients enrolled (27 females). Sixteen patients (30.2\%) were positive for anxiety symptoms, ten (18.9\%) for depressive symptoms and five (9.4\%) for anxiety-depressive symptoms. The perceived QoL was precarious in twelve subjects (22.2\%): eight $(15.9 \%)$ had low score $(\leq 42)$ at "Mental Component Summary" (MCS) subscale, three (5.7\%) at the "Mental Health" item and one patient $(1.9 \%)$ at the "Vitality" one. At the multiple regression analysis, depressive $(\mathrm{OR}=28.63 ; P=0.01)$ and anxiety-depressive symptoms $(\mathrm{OR}=11.16 ; P=0.02)$ were associated with MCS.
Conclusions The association emerging from the present study between depressive/anxiety symptoms and the MCS component of SF36 is consistent with available literature. Study design and small sample size do not allow to generalize results, that need further studies to be confirmed.

Disclosure of interest The authors have not supplied their declaration of competing interest.

http://dx.doi.org/10.1016/j.eurpsy.2017.02.237

\section{EW0624}

\section{Prevalence, incidence and comparative meta-analysis of all-cause and specific-cause cardiovascular disease in patients with serious mental illness}

M. Solmi ${ }^{1,2,3, *}$, N. Veronese ${ }^{2,4}$, B. Beatrice ${ }^{2,5}$, R. Stella ${ }^{1}$, S. Paolo ${ }^{1}$, G. Davide ${ }^{1}$, E. Collantoni ${ }^{1}$, G. Pigato ${ }^{1}$, A. Favaro ${ }^{1}$, B. Stubbs ${ }^{6,7}$, A.F. Carvalho ${ }^{8}$, D. Vacampfort ${ }^{9,10}$,

C.U. Correll $11,12,13,14$

${ }^{1}$ University of Padua, Neuroscience Department, Padua, Italy

${ }^{2}$ Institute for clinical Research and Education in Medicine,

Neuroscience Department, Padua, Italy

${ }^{3}$ ULSS 17, Mental Health Department, Padua, Italy

4 University of Padua, Department of Medicine, Padua, Italy

${ }^{5}$ ULSS 10, Mental Health Department, Portogruaro, Italy

${ }^{6}$ South London and Maudsley NHS Foundation Trust, Physiotherapy Department, London, United Kingdom

7 King's College London, Health Service and Population Research Department- Institute of Psychiatry, Psychology and Neuroscience, London, United Kingdom

${ }^{8}$ Faculty of Medicine, Federal University of Ceará, Departmend of Clinical Medicine and Translational Psychiatry Research Group,

Fortaleza, Brazil

${ }^{9}$ KU Leuven, Department of Rehabilitation Sciences, Leuven, Belgium

${ }^{10}$ KU Leuven, University Psychiatric Center KU Leuven, Leuven, Belgium

11 The Zucker Hillside Hospital, Psychiatry Research, New York, USA

12 Albert Einstein College of Medicine, Medicine, New York, USA

${ }^{13}$ The Feinstein Institute for Medical Research, Research, New York, USA

${ }^{14}$ Hofstra Northwell School of Medicine, Medicine, New York, USA

* Corresponding author.

Patients with severe mental illness (SMI) have been described at higher risk of cardiovascular disease (CVD). The aim of this systematic review and meta-analysis was to quantify prevalence, incidence, cross-sectional association and longitudinal increased risk of coronary heart disease (CHD), stroke, transient ischemic attack and cerebrovascular disease (CBVD), heart failure (HF), peripheral vascular disease (PVD), death due to CVD, and any CVD in patients with SMI. We included 92 studies, with a total population of $3,371,461$ patients $(\mathrm{BD}=241,226, \mathrm{MDD}=476,102, \mathrm{SCZ}=1,721,586$, SMI $=932,547)$ and $113,925,577$ controls. Pooled prevalence of any CVD in SMI was 9.9\% (95\% CI=7.4-13.3) (33 studies, 360,144 patients). Compared to controls, after adjusting for a median of 7 confounders, SMI was associated with higher risk of CVD in crosssectional studies, OR:1.53 (95\% CI=1.27-1.83) (11 studies), with CHD OR: 1.51 (95\% CI=1.47-1.55) (5 studies), with CBVD OR: 1.42 (95\% CI $=1.21-1.66)$ ( 6 studies), and tended to be associated with $\mathrm{HF}$ OR: $1.28(95 \% \mathrm{CI}=0.99-1.65)$ ( 4 studies). Cumulative incidence was 3.6 CVD events in a median follow-up period of 8.4 years (range: 1.76-30). After considering a median of 6 confounders, SMI was associated with higher longitudinal risk of CVD in longitudinal studies HR: 1.78 (95\% CI = 1.6, 1.98) (31 studies), of CHD: HR: 1.54 (95\% CI 1.30-1.82) (18 studies), of CBVD HR: 1.64 (95\% CI 1.26-2.14) (11 studies), of HF HR:2.10 (95\% CI 1.64-2.70)(2 studies), of PVD, unadjusted RR: 3.11 (95\% CI 2.46-3.91) (3 studies), of death due to CVD, HR 1.85 (95\% CI 1.53-2.24) (16 studies). In this meta-analysis, the 